\title{
He Chengtian's Inequalities for a Coupled Tangent Nonlinear System Arisen in Packaging System
}

\author{
Jun Wang, ${ }^{1}$ Zhi-geng Fan, ${ }^{2}$ Li-xin Lu, ${ }^{1}$ An-jun Chen, ${ }^{1}$ and Zhi-wei Wang ${ }^{3}$ \\ ${ }^{1}$ Jiangsu Province Key Laboratory of Advanced Food Manufacturing Equipment and Technology, \\ Department of Packaging Engineering, Jiangnan University, Wuxi 214122, China \\ ${ }^{2}$ School of Light Industry, Zhejiang University of Science and Technology, Hangzhou 310023, China \\ ${ }^{3}$ Key Laboratory of Product Packaging and Logistics of Guangdong Higher Education Institutes, Jinan University, Zhuhai 519070, China
}

Correspondence should be addressed to Jun Wang; wangj_1982@jiangnan.edu.cn and Zhi-wei Wang; wangzw@jnu.edu.cn

Received 19 July 2013; Accepted 27 August 2013

Academic Editor: Guo-Cheng Wu

Copyright (C) 2013 Jun Wang et al. This is an open access article distributed under the Creative Commons Attribution License, which permits unrestricted use, distribution, and reproduction in any medium, provided the original work is properly cited.

He Chengtian's inequalities from ancient Chinese algorithm are applied to strong tangent nonlinear packaging system. The approximate solution is obtained and compared with the solution yielded by computer simulation, showing a great high accuracy of this method. The suggested approach provides a novel method to solve some essential problems in packaging engineering.

\section{Introduction}

In order to avoid some restrictions of perturbation method [1], some other methods are developed, such as the homotopy perturbation method (HPM) [2,3], the variational iteration method (VIM) [4-6], the homotopy analysis method (HAM) [7], and He Chengtian's inequalities which cannot be found in the literature but recently reported in [8]. The max-min approach which is developed from the idea of ancient Chinese mathematics is demonstrated to be of convenient application, less calculation, and high accuracy. Among current researches about He Chengtian's inequalities and their applications [9-11], few involved coupled nonlinear problems. In our previous research [12], He Chengtian's inequalities were introduced to study the nonlinear dropping shock response for coupled cubic nonlinear packaging system, showing the effectiveness of the method. In packaging system, many cushioning materials behave as the tangent nonlinear characteristics $[13,14]$, and the dropping shock response of tangent packaging system with critical component is also studied [15]. In this paper, He Chengtian's inequalities are applied to the coupled nonlinear tangent packaging system with critical component, and the obtained analytical solution is compared with the solution of computer simulation. The aim of this research is to suggest a new and simple mathematical method for solving the nonlinear dropping shock equations arisen in packaging system.

\section{Modelling and Equations}

The governing equations of tangent nonlinear cushioning packaging system with the critical component can be expressed as [15]

$$
\begin{gathered}
m_{1} x^{\prime \prime}+k_{1}(x-y)=0, \\
m_{2} y^{\prime \prime}+\frac{2 k_{2} d_{b}}{\pi} \tan \frac{\pi}{2 d_{b}} y-k_{1}(x-y)=0,
\end{gathered}
$$

where

$$
\begin{gathered}
x(0)=0, \\
y(0)=0, \\
x^{\prime}(0)=\sqrt{2 g h}, \\
y^{\prime}(0)=\sqrt{2 g h .}
\end{gathered}
$$

Here the coefficients $m_{1}$ and $m_{2}$ denote the mass of the critical component and the main part of the product, 
respectively, while $k_{1}$ and $k_{2}$ are the coupling stiffness of the critical component and that of cushioning pad, respectively, $d_{b}$ is the compression limit of the cushioning pad, and $h$ is the dropping height. Equation (1) can be equivalently written in the following forms:

$$
\begin{gathered}
\ddot{X}+\omega_{1}^{2}(X-Y)=0 \\
\ddot{Y}+Y+\frac{1}{3} Y^{3}+\frac{2}{15} Y^{5}+\left(1-\omega_{2}^{2}\right)(X-Y)=0,
\end{gathered}
$$

where

$$
\begin{gathered}
X=\frac{x}{\sqrt{2 d_{b} / \pi}}, \\
Y=\frac{y}{\sqrt{2 d_{b} / \pi}}, \\
\tau=\frac{t}{\sqrt{m_{2} / k_{2}}}, \\
\omega_{1}=\sqrt{\frac{k_{1} m_{2}}{k_{2} m_{1}}}, \\
\omega_{2}=\sqrt{1+\frac{m_{1}}{m_{1}^{2}}}, \\
X(0)=0, \\
\dot{X}(0)=0, \\
\dot{X}(0)=\frac{\sqrt{m_{2} / k_{2}}}{\sqrt{2 d_{b} / \pi}} \sqrt{2 g h,} \\
\sqrt{m_{2} / k_{2}} \sqrt{2 d_{b} / \pi} \sqrt{2 g h .}
\end{gathered}
$$

\section{Application of He Chengtian's Inequalities}

From (3), we can easily obtain

$$
\begin{aligned}
Y^{(4)} & +\left(\omega_{1}^{2}+\omega_{2}^{2}+Y^{2}+\frac{2}{3} Y^{4}\right) \ddot{Y} \\
& +\omega_{1}^{2}\left(Y+\frac{1}{3} Y^{3}+\frac{2}{15} Y^{5}\right)=0 .
\end{aligned}
$$

Rewrite (13) in the form

$$
\begin{aligned}
Y^{(4)}=- & {\left[\left(\frac{\omega_{1}^{2}+\omega_{2}^{2}}{Y}+Y^{2}+\frac{2}{3} Y^{3}\right) \ddot{Y}\right.} \\
& \left.+\omega_{1}^{2}\left(1+\frac{1}{3} Y^{2}+\frac{2}{15} Y^{4}\right)\right] Y .
\end{aligned}
$$

According to He Chengtian's inequalities, we choose a trialfunction in the form

$$
Y=A \sin (\Omega \tau)
$$

which meets the initial conditions as described in (10) and (12).

By simple analysis, from (14)-(15), we know that

$$
\begin{aligned}
\Omega^{4}= & \left(\omega_{1}^{2}+\omega_{2}^{2}\right) \Omega^{2}-\omega_{1}^{2} \\
& +\left(A^{2} \Omega^{2} \sin ^{2} \Omega \tau+\frac{2}{3} A^{4} \Omega^{2} \sin ^{4} \Omega \tau\right) \\
& -\left(\frac{1}{3} A^{2} \omega_{1}^{2} \sin ^{2} \Omega \tau+\frac{2}{15} A^{4} \omega_{1}^{2} \sin ^{4} \Omega \tau\right) .
\end{aligned}
$$

The maximal and minimal value of $\sin ^{2} \Omega \tau$ are, respectively, 1 and 0 . So, we can immediately obtain

$$
\begin{aligned}
f_{\min } & =\left(\omega_{1}^{2}+\omega_{2}^{2}+A^{2}+\frac{2}{3} A^{4}\right) \Omega^{2}-\frac{2 A^{4}+5 A^{2}+15}{15} \omega_{1}^{2} \\
& <\Omega^{4}<\left(\omega_{1}^{2}+\omega_{2}^{2}\right) \Omega^{2}-\omega_{1}^{2}=f_{\max } .
\end{aligned}
$$

According to He Chengtian's interpolation [8, 12], we obtain

$$
\Omega^{4}=\frac{m f_{\min }+n f_{\max }}{m+n}=\left(\omega_{1}^{2}+\omega_{2}^{2}\right) \Omega^{2}-\omega_{1}^{2}+k M,
$$

where $m$ and $n$ are weighting factors, $k=m /(m+n)$, and $M=\left(A^{2}+(2 / 3) A^{4}\right) \Omega^{2}-\left(\left(2 A^{4}+5 A^{2}\right) / 15\right) \omega_{1}^{2}$.

Then, the approximate solution of (13) can be written as

$$
Y=A \sin \left[\left(\omega_{1}^{2}+\omega_{2}^{2}\right) \Omega^{2}-\omega_{1}^{2}+k M\right]^{1 / 4} \tau .
$$

To determine the value of $k$, substituting (19) into (13) results in the following residual [8]:

$$
R(\tau, k)=\left(\Omega^{2}-\frac{1}{3} \omega_{1}^{2}\right) Y^{3}+\left(\frac{2}{3} \Omega^{2}-\frac{2}{15} \omega_{1}^{2}\right) Y^{5}-k M Y .
$$

And by setting

$$
\int_{0}^{T / 4} R(\tau, k) \sin \Omega \tau d \tau=0,
$$

where $T=2 \pi / \Omega$, we obtain the $k$ value as

$$
k=\frac{6 A^{2}\left(\Omega^{2}-(1 / 3) \omega_{1}^{2}\right)+5 A^{4}\left((2 / 3) \Omega^{2}-(2 / 15) \omega_{1}^{2}\right)}{8 M} .
$$

Substituting (22) into (18) yields

$$
\begin{aligned}
8 \Omega^{4}= & 8\left(\omega_{1}^{2}+\omega_{2}^{2}\right) \Omega^{2}-8 \omega_{1}^{2}+6 A^{2}\left(\Omega^{2}-\frac{1}{3} \omega_{1}^{2}\right) \\
& +5 A^{4}\left(\frac{2}{3} \Omega^{2}-\frac{2}{15} \omega_{1}^{2}\right) .
\end{aligned}
$$

From (23), we can easily obtain the frequency value $\Omega$. Table 1 gives the values of $\Omega$ with different $\omega_{1}$ and $\omega_{2}$, and Figure 1 shows that the approximate solution, (19), agrees well with the exact solution for various different values of $\omega_{1}$ and $\omega_{2}$, where the initial velocity is assumed as $\dot{Y}(0)=A \Omega=1$. 


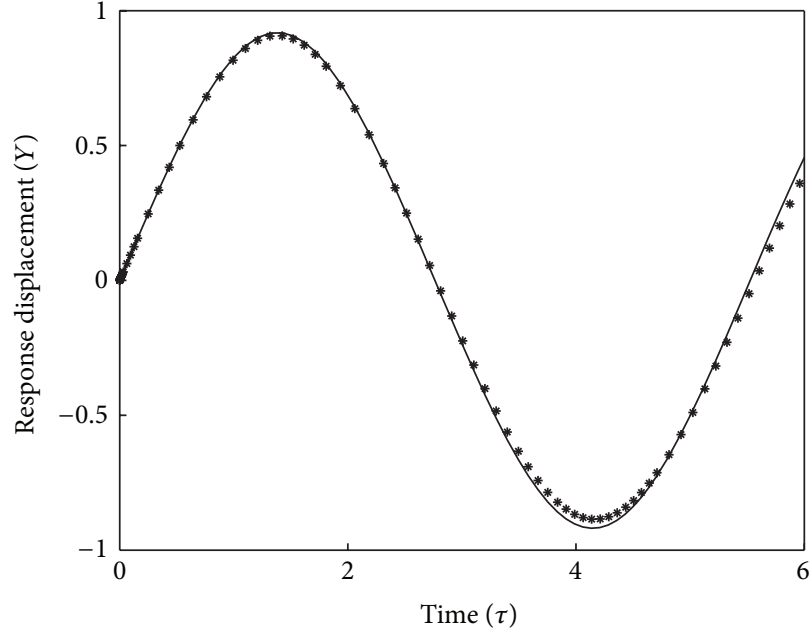

(a) $\omega_{1}=3, \omega_{2}=1$

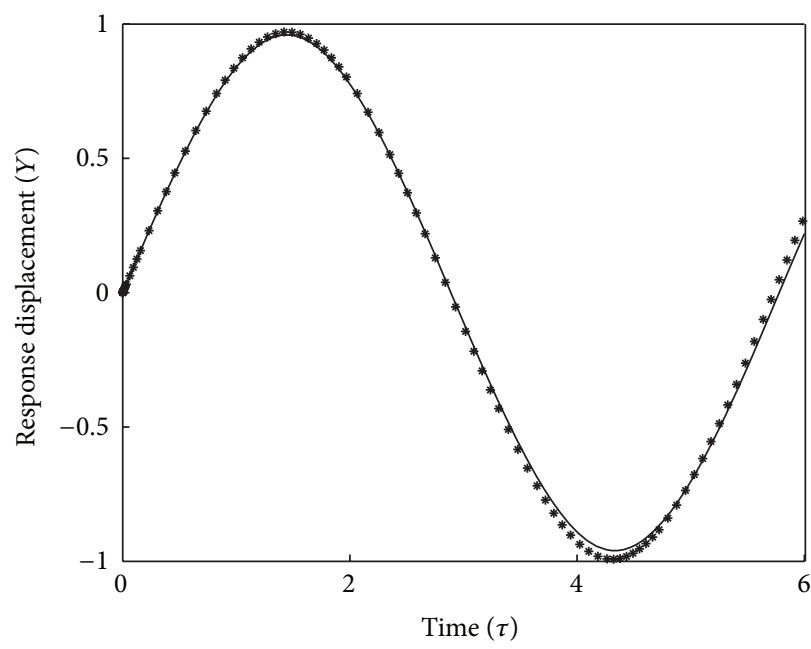

(c) $\omega_{1}=5, \omega_{2}=2$

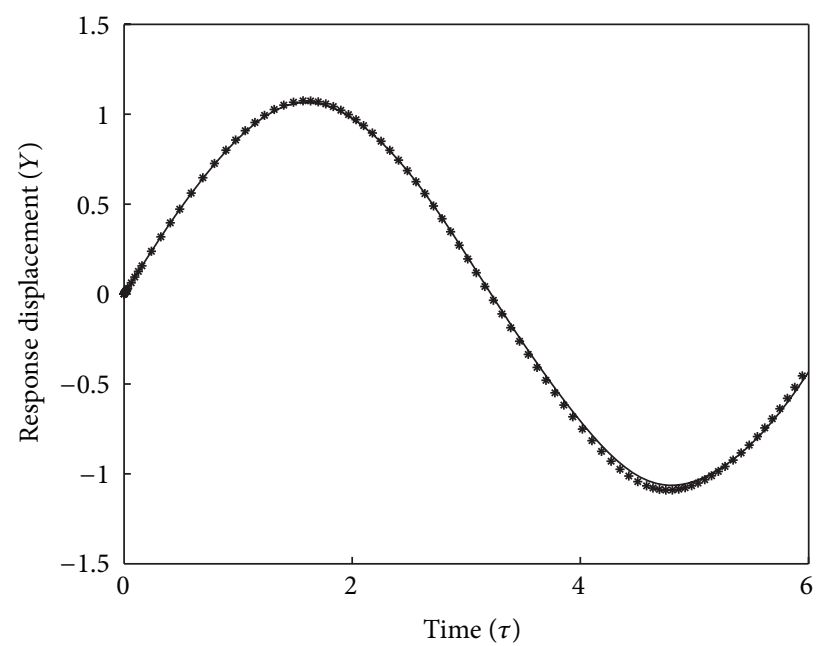

(b) $\omega_{1}=4, \omega_{2}=3$

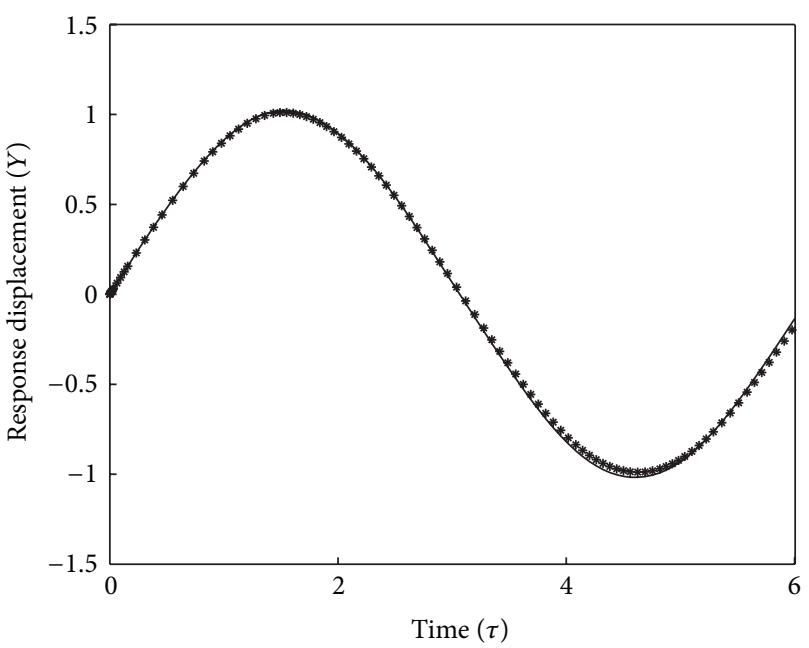

(d) $\omega_{1}=5, \omega_{2}=3$

FIGURE 1: Comparison of the approximate solution with the exact solution (asterisk: the approximate solution; continuous line: the exact solution).

TABLE 1: Values of $\Omega$ from (23) with different values of $\omega_{1}$ and $\omega_{2}$.

\begin{tabular}{cccc}
\hline$\omega_{1}$ & & $\omega_{2}$ & \\
& 1 & 2 & 3 \\
\hline 3 & 1.08323559 & 0.95968791 & 0.84368664 \\
4 & 1.10039777 & 1.02416608 & 0.93503514 \\
5 & 1.10757043 & 1.05683775 & 0.99009216 \\
\hline
\end{tabular}

\section{Conclusion}

He Chengtian's inequalities are for the first time applied to study the nonlinear response of coupled tangent packaging system. The results show that this method can be easily used in engineering application with high accuracy without cumbersome calculation.

\section{Acknowledgments}

The authors would like to appreciate the financial support of National Natural Science Foundation of China (Grant no. 51205167), Research Fund for the Doctoral Program of Higher Education of China (Grant no. 20120093120014), the Open Fund of Key Laboratory of Product Packaging and Logistics of Guangdong Higher Education Institutes, Jinan University, and the Fundamental Research Funds for the Central Universities (Grant no. JUSRP51302A).

\section{References}

[1] A. H. Nayfeh, Perturbation Methods, Wiley-VCH, Weinheim, Germany, 2007.

[2] A. A. Elbeleze, A. Kılıçman, and B. M. Taib, "Homotopy perturbation method for fractional black-scholes European option pricing equations using Sumudu transform," Mathematical 
Problems in Engineering, vol. 2013, Article ID 524852, 7 pages, 2013.

[3] S. Das and P. K. Gupta, "Approximate analytical solutions of time-space fractional diffusion equation by Adomian decomposition method and homotopy perturbation method," Communications in Fractional Calculus, vol. 2, no. 1, pp. 29-35, 2011.

[4] G. C. Wu, "New trends in the variational iteration method," Communications in Fractional Calculus, vol. 2, no. 2, pp. 59-75, 2011.

[5] H. Jafari and C. M. Khalique, "Homotopy perturbation and ariational iteration methods for solving fuzzy differential equations," Communications in Fractional Calculus, vol. 3, no. 1, pp. 38-48, 2012.

[6] G. C. Wu and D. Baleanu, "New applications of the variational iteration method-from differential equations to q-fractional difference equations," Advances in Difference Equations, vol. 2013, p. 21, 2013.

[7] S. J. Liao, Beyond Perturbation: Introduction to Homotopy Analysis Method, Chapman \& Hall/CRC, New York, NY, USA, 2003.

[8] J. H. He, "Solution of nonlinear equations by an ancient Chinese algorithm," Applied Mathematics and Computation, vol. 151, no. 1, pp. 293-297, 2004.

[9] R. Azami, D. D. Ganji, H. Babazadeh, A. G. Dvavodi, and S. S. Ganji, "He's max-min method for the relativistic oscillator and high order duffing equation," International Journal of Modern Physics B, vol. 23, no. 32, pp. 5915-5927, 2009.

[10] S. A. Demirbağ and M. O. Kaya, "Application of He's max-min approach to a generalized nonlinear discontinuity equation," International Journal of Nonlinear Sciences and Numerical Simulation, vol. 11, no. 4, pp. 269-272, 2010.

[11] S. S. Ganji, D. D. Ganji, A. G. Davodi, and S. Karimpour, "Analytical solution to nonlinear oscillation system of the motion of a rigid rod rocking back using max-min approach," Applied Mathematical Modelling, vol. 34, no. 9, pp. 2676-2684, 2010.

[12] J. Wang, "He’s Max-Min approach for coupled cubic nonlinear equations arising in packaging system," Mathematical Problems in Engineering, vol. 2013, Article ID 382509, 4 pages, 2013.

[13] Z. W. Wang, "Dropping damage boundary curves for cubic and tangent package cushioning systems," Packaging Technology and Science, vol. 15, no. 5, pp. 263-266, 2002.

[14] D. Gao and F. D. Lu, "Shock response of a nonlinear tangent packaging system with rotation," Journal of Vibration and Shock, vol. 29, no. 10, pp. 131-210, 2010.

[15] J. Wang, J. Jiang, L. Lu, and Z. Wang, "Dropping damage evaluation for a tangent nonlinear system with a critical component," Computers and Mathematics with Applications, vol. 61, no. 8, pp. 1979-1982, 2011. 


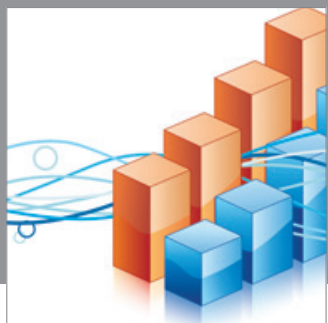

Advances in

Operations Research

mansans

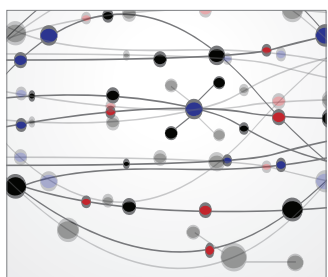

The Scientific World Journal
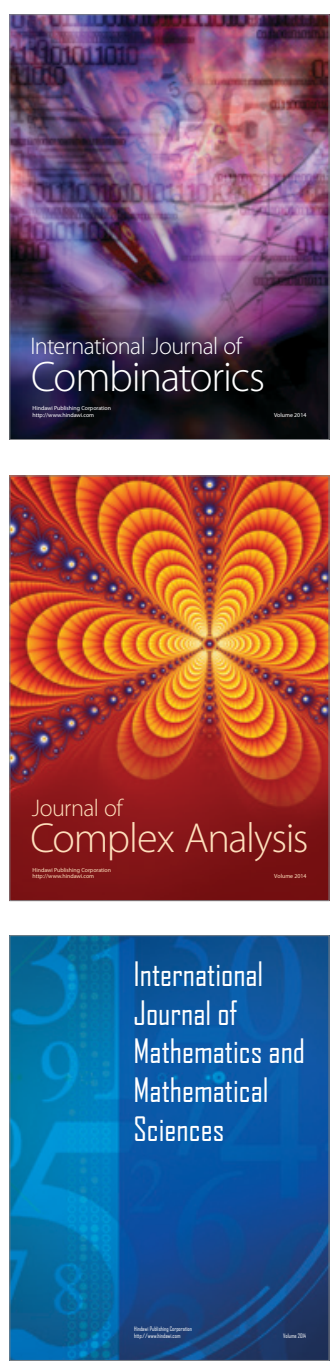
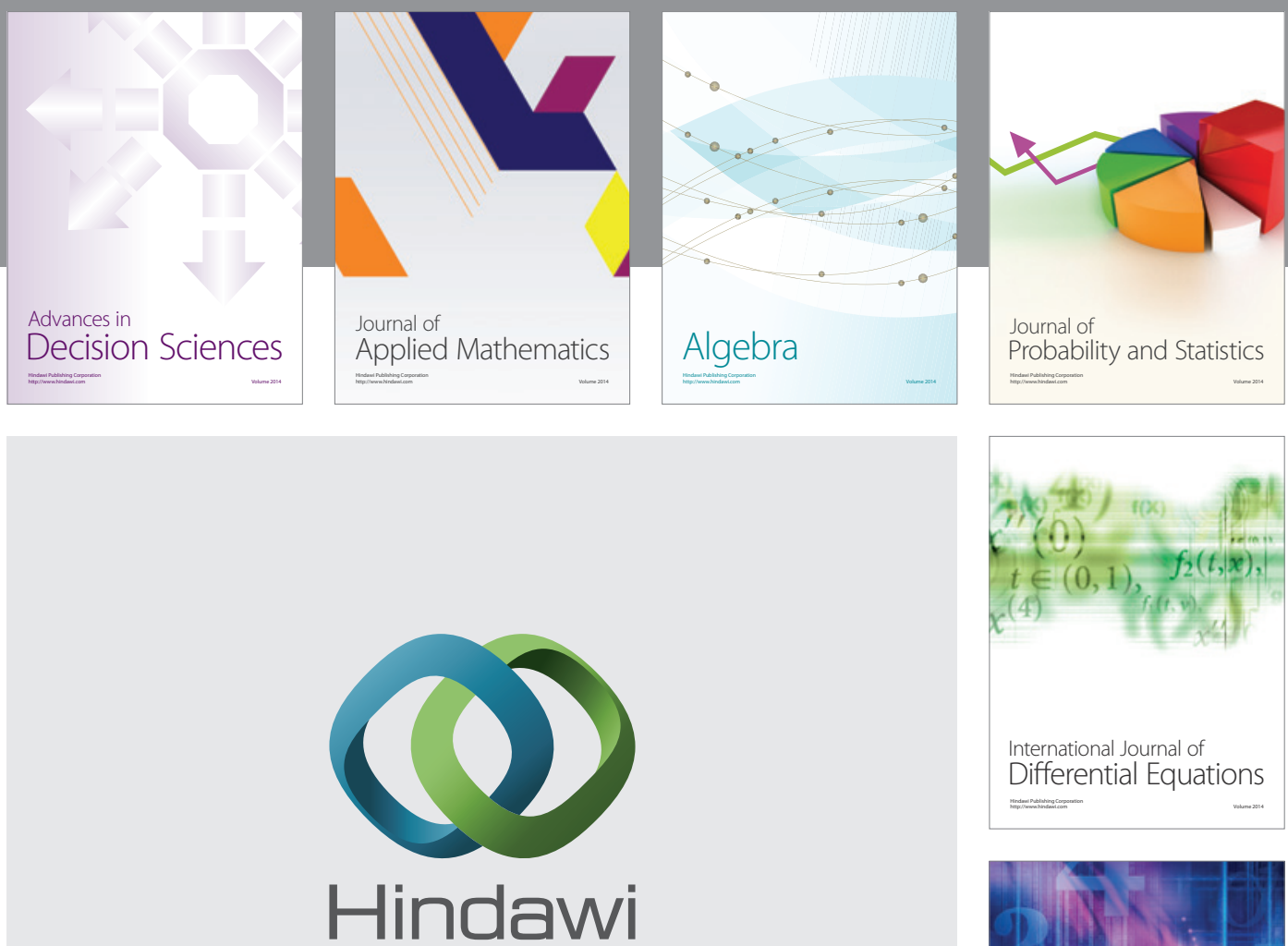

Submit your manuscripts at http://www.hindawi.com
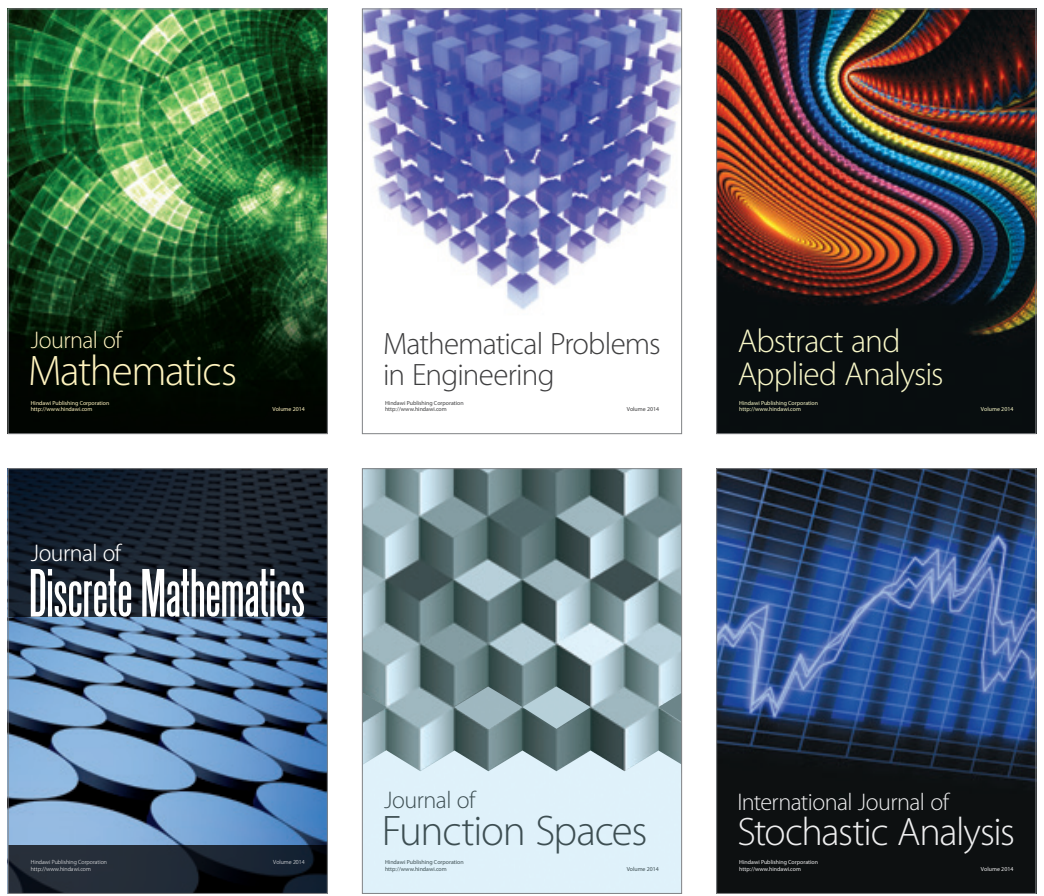

Journal of

Function Spaces

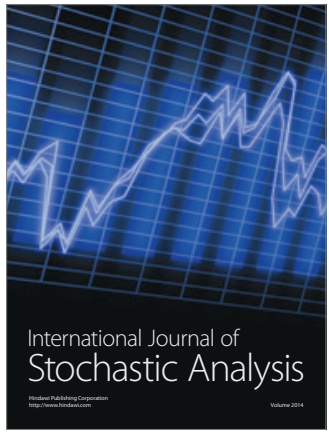

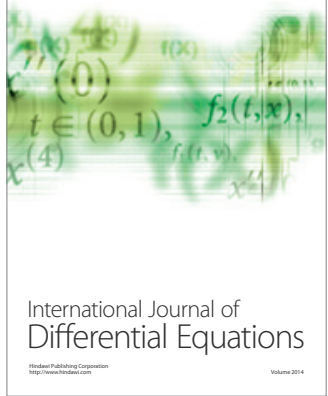
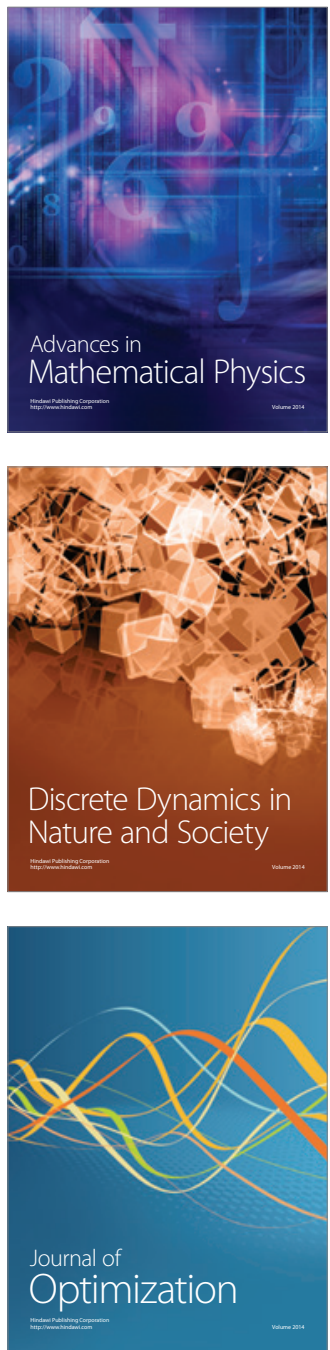